DOI: 10.17234/SRAZ.65.51

\title{
Regolarmente irregolare? Sull'allomorfia in verbi italiani e francesi altamente frequenti
}

\author{
Sabine Heinemann \\ Karl-Franzens-Universität Graz \\ sabine.heinemann@uni-graz.at
}

\begin{abstract}
Studi specialmente in ambito germanistico mostrano che l'irregolarità nei paradigmi di verbi ausiliari, modali o di movimento elementare riporta al concetto di rilevanza proposta da Bybee (1985). Questo concetto mostra una chiara correlazione fra sviluppo morfologico e frequenza token; invece, la frequenza type pare essere responsabile dell'adozione di schemi morfologici. Il contributo esamina i risultati di Nübling (2000) e l'applicabilità del concetto di rilevanza con particolare riferimento ai verbi fr. aller/it. andare, fr. venir/it. venire e fr. ant. ester (vs. estre > fr. mod. être)/it. stare, tenendo conto degli sviluppi avvenuti dall'epoca latina ad oggi.
\end{abstract}

Parole chiave: morfologia, allomorfia, analogia, irregolarità, frequenza

\section{Introduzione}

Di norma, i verbi che mostrano allomorfia dovuta a sviluppi fonetici riprendono, nel corso della storia, una forma regolare grazie all'analogia intraparadigmatica; tuttavia, come nel caso di elementi con una token frequency molto alta, ${ }^{1}$ se questi non vengono successivamente eliminati provocano irregolarità; allomorfia può essere causata anche da indebolimenti irregolari, come le assimilazioni o la caduta di interi segmenti. Sono tipiche, inoltre, analogie di differenziazione che si basano su singole forme del paradigma di altri verbi altamente frequenti e portano a distinzioni intraparadigmatiche. A livello interparadigmatico, diventano così visibili cluster o somiglianze di famiglia tra verbi con un massimo di frequenza d'uso (si veda p. es. it. ant. fo, vo su ho). Contrariamente, per verbi meno frequenti, normalmente si ha l'analogia di più forme del paradigma con classi di alta type frequency il che permette una

1 Secondo vari studi nell'ambito dell'approccio basati sull'uso (usage-based), la token frequency risulta essere il fattore più importante per lo sviluppo irregolare, anche se vengono discussi fattori come la salienza fonologica, la complessità morfonologica, l'interazione di forme irregolari con forme default o la semantica (con riguardo ad un eventuale cluster), per nominare solo alcuni (cf. p.es. Nübling 2000; Versloot/Adamdzyk 2018). 
regolarizzazione e un alto grado di coerenza. ${ }^{2}$ È possibile anche una distribuzione di allomorfi cambiata che sembra riflettere un modello o uno schema sottostante. ${ }^{3}$ Inoltre, anche un cambiamento lessicale può essere fonte di irregolarità, così il suppletivismo totale provoca una distinzione massima all' interno del paradigma. ${ }^{4}$ Infine, l'uso in costruzioni perifrastiche condiziona un aumento di frequenza drastico dei rispettivi verbi che spesso si traduce in un'espressione abbreviata. I casi prototipici di irregolarità e regolarità possono essere quindi concepiti come poli estremi di un continuum di (ir)regolarità morfologica. Fattori importanti sono la derivabilità di regole per l'allomorfia, ${ }^{5}$ la formazione di gruppi, la produttività, ${ }^{6}$ la somiglianza formale di singole radici e la trasparenza morfosemantica.

Tipicamente, sono irregolari e altamente frequenti ausiliari, verbi che fungono da copula, verbi elementari di movimento, di stato, verba dicendi e di percezione. Pertanto, nel seguente paragrafo si accennerà ad alcuni tentativi di spiegazione, mentre in quello successivo si cercherà di sintetizzare alcune osservazioni svolte sui verbi fr. aller/it. andare, fr. venir/it. venire e fr. ant. ester e estre ( $>$ fr. mod. etre)/ it. stare (con riferimento alla sola varietà standard). ${ }^{7}$

\section{Tentativi di spiegazione}

Il concetto di rilevanza proposto da Bybee (1985) risulta di massima importanza quando si parla di irregolarità morfologica; seppur abbia subito alcune modifiche nelle più recenti pubblicazioni, questo concetto è rimasto inalterato nelle assunzioni di base, abbinando le rispettive categorie e i loro modi di espressione. Il grado di rilevanza delle categorie flessive verbali per le lingue romanze può essere raffigurato in modo scalare:

2 Per il concetto di analogia si rinvia a Fertig 2016 e Bybee 2010: $93 f$.

3 Tali modelli, schemi o morfomi (nel senso di Maiden) fungono da principio paradigmatico per organizzare l'allomorfia presente in modo uniforme e rendere la memorizzazione più facile, perché alternanze fonologicamente diverse seguano lo stesso modello astratto; Maiden argomenta su base formale, senza prendere in considerazione eventuali motivi funzionali (Dammel 2010: 513; Maiden 2018). Ciò nonostante, questi schemi possono proteggere strutture allomorfiche e attirare nuovi verbi (si veda anche Nübling 2016: 288s.; Bybee 201: 102).

4 Un forte suppletivismo non è causato da un eventuale difettività del paradigma, ma piuttosto dalla necessità di differenziazione (cf. Nübling 2016: 286).

5 L'allomorfia dovuta a sviluppi fonetici è più regolare di quella basata su sviluppi morfologici.

6 Tra i fattori che determinano la produttività sono particolarmente importanti la type frequency e il grado di schematicity (nel senso di unità mentalmente differenziabili).

7 Per un trattamento molto più dettagliato della tematica si rinvia a Heinemann in corso di stampa. 


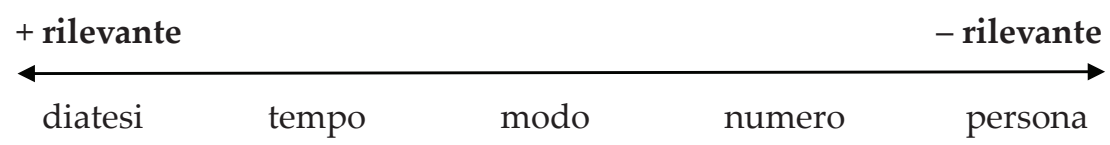

Fig. 1: Gerarchia di rilevanza delle categorie flessive verbali (adattato da Nübling 2000: 222) $)^{8}$

La diatesi è dunque altamente rilevante, in quanto rappresenta un cambiamento di prospettiva nella descrizione di un'azione; altrettanto importante è l'aspetto, il quale riflette il procedimento interno della stessa. Per contro, la categoria di tempo serve "solo" al riferimento temporale, ma riveste comunque un alto grado di rilevanza, per cui viene spesso trasferita nella radice. ${ }^{9}$ A causa della loro bassa rilevanza, numero e persona vengono spesso espressi non solo con flessivi, ma anche sintatticamente (soggetti clitici). Ora, una token frequency molto alta porta all'espressione nella radice anche di categorie meno rilevanti, provoca cioè una drift a sinistra, e questo diventa importante per l'incremento di allomorfia nel gruppo di verbi qui considerati. ${ }^{10} \mathrm{~L}$ 'aumento di irregolarità non è però un processo teleologico, ma risulta da singole ottimizzazioni governate sia dalla rilevanza che dalla frequenza (Nübling/Dammel 2004, 203f.). Werner (1987) integra l'irregolarità legata all'alta frequenza d'uso nel suo modello di economia linguistica e accenna alla mescolanza di brevità e differenziazione spesso riscontrabile all'interno dei paradigmi. In questi casi all'utente conviene memorizzare le forme complesse ${ }^{11}$ che così risultano facilmente accessibili, mentre per le forme meno frequenti è più appagante la memorizzazione delle regole di formazione per dar vita alle voci nel momento dell'enunciato.

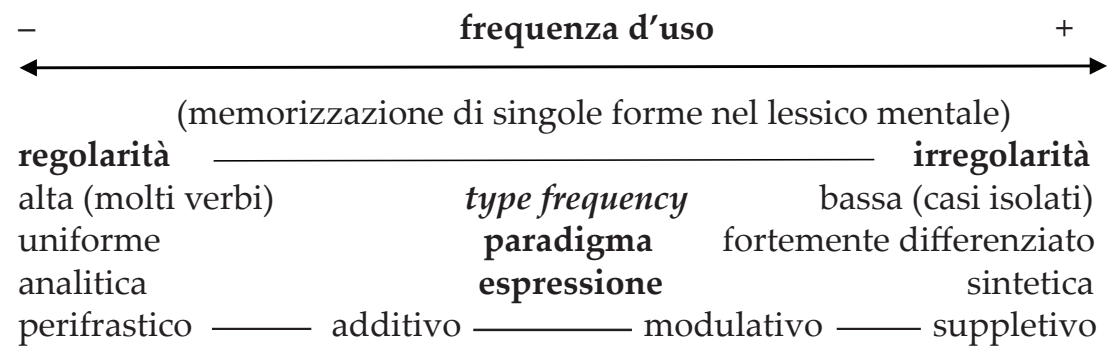

Fig. 2: Livelli di compressione (adattato da Werner 1987: 300)

8 Rilevanza qui si riferisce all'influsso semantico delle categorie sul concetto di base e dunque del verbo.

9 Per le lingue romanze si nota una chiara distinzione delle radici in dipendenza della categoria tempo soprattutto con riferimento alle radici suppletive (cf. anche Veselinova 2006: 175s.). Una distinzione così netta non si mostra per l'allomorfia non-suppletiva; tuttavia, si possono delineare morfomi nel senso di Maiden (p. es. 2018).

10 È da tener presente però la diversa frequenza delle singole forme - così la terza persona singolare è molto più frequente delle prime due, le terze persone coprono il 60-70\% delle forme. Per il numero è dominante il singolare, per la categoria del tempo predomina nettamente il presente, per il modo e per la diatesi sono l'indicativo e l'attivo a essere i membri più frequenti.

11 Per i vari approcci cf. Bybee 1985: 111-125; Pinker 1999; Booij 2007. 


\section{Allomorfia verbale in francese e italiano}

Come detto nel paragrafo introduttivo, tra i verbi irregolari troviamo quelli di movimento e di stato, come fr. aller/it. andare, fr. venir/it. venire e fr. ant. ester (vs. estre $>$ fr. mod. être)/it. stare. Più delle altre, la prima coppia è contrassegnata da un suppletivismo vistoso - in latino IRE dispone di una radice molto breve (in alcune forme [i]) che rappresenta un motivo essenziale per l'eteroclisia. ${ }^{12}$ Le forme del congiuntivo presente o anche del participio presente (EAM, IĒNS) Stanno alla base di forme con consonantizzazione in italiano antico (giamo ecc. da cui per estensione gire), portando così ad una variazione delle radici in gi- e $i$-. ${ }^{13}$ Mentre in francese IRE si conserva nelle forme del futuro e del condizionale, in italiano subentrano forme con *and-e vad- (quest'ultimo predominante nel paradigma più frequente, cioè l'indicativo presente). Quindi, benché suppletivismo significhi un massimo di differenziazione formale, anche per i verbi frequenti non è garantito il suo mantenimento. Per un paradigma meno frequente come il congiuntivo presente si ha un forte sincretismo per le forme rizotoniche sia in italiano che in francese, mentre la distinzione delle forme dell'indicativo risulta chiara anche per il francese, ${ }^{14}$ ed è dovuta in parte a intrecci con altri paradigmi - così alcune forme del francese (antico) come vai, vas e vat mostrano l'influsso di aveir (fr. mod. avoir), vait può essere anche analogo a fait di faire. Vont a sua volta è regolare, ma probabilmente è alla fonte di fr. ant. estont (ester). Infine, anche il passé simple del paradigma è diventato irregolare (in latino vigeva un perfetto di tipo debole).

Nonostante l'abolizione delle forme basate su $(g)$ ire, in italiano si preserva il suppletivismo di vad-e and-nel presente indicativo anche se vad- è relativo al solo presente (anche al congiuntivo per le forme rizotoniche come pure per l'imperativo si ha $v a(d)$-). Come per il francese le forme dell'indicativo presente mostrano una sovrapposizione con quelle di avere (con vo (it. mod. vado), vai, va, vanno).

VENIRE in latino è abbastanza regolare, mentre in epoca medievale aumenta l'irregolarità; irregolarità che si è conservata fino al francese moderno e dovuta principalmente al dittongamento e alla nasalizzazione (con successiva denasalizzazione a seconda del contesto sillabico nel francese medio). Altri allomorfi radicali si trovano nel futuro in $v(i) e n d r a$, con la forma dittongata analoga con le forme rizotoniche dell'indicativo presente ((il) vient). Gli sviluppi più complessi si rivelano nel paradigma del perfetto, dove si trovano sia forme regolari (come quella della prima persona singolare vin dovuta a metafonia) che

12 Per le singole forme si rinvia a Buridant (2000) e Penello et al. (2010).

13 Già in italiano antico però non si hanno più forme a base di IRE in tutti i paradigmi; dove ce ne sono, sono o esclusive o varianti di quelle con and-. Ne risulta che il suppletivismo non serve a colmare lacune nel paradigma, ma a sostituire altre forme esistenti; questo si vede anche nel cambiamento nella perifrasi grammaticalizzata $(g)$ ire + gerundio (si vedano esempi come giva andando p. es. in Boccaccio) in andare + gerundio.

14 Questo sviluppo riflette la rispettiva forza lessicale delle singole forme (lexical strength, Bybee 1985: 117s.) o autonomia. 
analogiche (con vins e vint per la seconda e terza persona su vin della prima). La variante della seconda persona, fr. ant. venis, è regolare e funge da base per le forme arizotoniche del plurale (venismes e venistes). Per il congiuntivo presente, si ha un'analogia rispetto a valeir, un importante modello anche per aller. Le analogie intra- e interparadigmatiche condizionano alcuni livellamenti; tuttavia, si mantiene l'allomorfia nel presente indicativo e nel perfetto. Anche l'italiano (moderno e antico) mostra un'allomorfia radicale forte, specie per l'indicativo presente, arricchita da venn- nel perfetto e da verr- nel futuro e nel condizionale. Per un'analogia interparadigmatica (cf. piangere - piango), le forme regolari con palatalizzazione dell'italiano antico (vegno) vengono sostuite da quelle con velare. Le forme del perfetto mostrano la tipica distribuzione delle radici con venni e seguono lo sviluppo del perfetto latino in -ur; infine, nelle forme rizotoniche si trova l'allomorfo dell'infinito, cioè ven-.

Rispetto a lat. STARE è interessante il duplice risultato ottenuto nelle lingue considerate: mentre in italiano ha proseguito sulla strada del verbo statico di luogo, per l'imperfetto in francese si osserva, già per la fase antica, l'entrata di ester nel paradigma di estre (< lat. volg. ESSERE); ed è probabile che questa formi anche la radice del futuro e del condizionale. Il paradigma di fr. mod.être rispecchia un suppletivismo favorito non solo dalla semantica ma in questo caso anche dalla vicinanza formale dei due verbi. Tuttavia, diversamente da aller, il suppletivismo sottostante al paradigma dal parlante odierno normalmente non viene percepito. Circa l'italiano stare, non sono interessanti solo i vari intrecci interparadigmatici con altri verbi altamente frequenti (si veda ad esempio l'analogia con avere per le forme del presente indicativo); è interessante anche lo sviluppo di un nuovo paradigma per il perfetto, dovuto anche agli influssi reciproci tra stare e dare benché la forma stetti è regolarmente sviluppata (su base del lat. volg. * ${ }^{\text {STETUI), per }}$ varie analogie ed estensioni si arriva alla formazione del perfetto tramite -etti/-ei.

Riassumendo, malgrado la forte allomorfia in epoca medievale, soprattutto in francese si nota una riduzione della marca flessiva specie di persona, cosa che condiziona l'omofonia di varie forme (fr. mod. vas, va [va], viens, viens, vient [vjẽ]); questo anche se la tendenza al sincretismo è molto più spiccata nei verbi regolari. In ogni caso i vari sviluppi avvenuti nella fase medievale rivelano la validità del concetto di rilevanza legata alla frequenza d'uso.

\section{Bibliografia}

Booij, Geert (22007). The Grammar of Words. An Introduction to Linguistic Morphology, Oxford: University Press.

Buridant, Charles (2000). Nouvelle grammaire de l'ancien français, Paris: Sedes.

Bybee, Joan (1985). Morphology. A study of the relation between meaning and form, Amsterdam/Philadelphia: Benjamins.

Bybee, Joan (2010). Language Change, Cambridge: University Press.

Dammel, Antje (2010). Wenn die 2. Person mit der 3. Person - Wechselflexion kontrastiv: Deutsch - Luxemburgisch - Westfriesisch - Faröisch, in: Konstras- 
tive Germanistische Linguistik, Teilband 1 [a cura di Antje Dammel / Sebastian Kürschner / Damaris Nübling], Hildesheim/Zürich/New York: Olms, pp. 473-522.

Fertig, David (2016). Mechanisms of paradigm leveling and the role of universal preferences in morphophonological change, in: Diachronica, 33, pp. 423-460.

Heinemann, Sabine (in corso di stampa). Hochfrequenz und Irregularität - zur Stammallomorphie bei (a)frz. al(l)er/(a)it. andare, (a)frz. venir/(a)it. venire und (a)frz. ester/(a)it. stare", in: Zeitschrift für romanische Philologie.

Maiden, Martin (2018). The Romance Verb. Morphomic Structure and Diachrony, Oxford: University Press.

Nübling, Damaris (2000). Prinzipien der Irregularisierung. Eine kontrastive Analyse von zehn Verben in zehn germanischen Sprachen, Tübingen: Niemeyer.

Nübling, Damaris/Dammel, Antje (2004). Relevanzgesteuerter morphologischer Umbau im Frühneuhochdeutschen, in: Beiträge zur Geschichte der deutschen Sprache und Literatur, 126 (2), pp. 177-207.

Nübling, Damaris (2016). Und sie leben doch: Zur Reorganisation starker Verben in germanischen Sprachen, in: PerspektivWechsel oder Die Wiederentdeckung der Philologie, vol. 1: Sprachdaten und Grundlagenforschung in der Historischen Linguistik [a cura di Sarah Kwekkeboom / Sandra Waldenberger], Berlin: Erich Schmidt, pp. 273-290.

Penello, Nicoletta/Benincà, Paola/Vanelli, Laura/Maschi, Roberta (2010). Morfologia flessiva, in: Grammatica dell'italiano antico, vol. 2 [a cura di Lorenzo Renzi / Francesco Salvi], Bologna: Il Mulino, pp. 1389-1491.

Pinker, Steven (1999). Words and Rules. The Ingredients of Language, London: Weidenfeld \& Nicolson.

Veselinova, Ljuba N. (2006). Suppletion in verb paradigms: Bits and pieces of the puzzle, Amsterdam/Philadelphia: Benjamins.

Versloot, Arjen P./Adamczyk, E. (2018). Plural inflection in North Sea Germanic languages. A multivariate analysis of morphological variation, in: Reorganising Grammatical Variation. Diachronic studies in the retention, redistribution and refunctionalisation of linguistic variants [a cura di Antje Dammel / Matthias Eitelmann / Mirjam Schmuck], Amsterdam/Philadelphia: Benjamins, pp. 17-55.

Werner, Otmar (1987). Natürlichkeit und Nutzen morphologischer Irregularität, in: Beiträge zum 3. Essener Kolloquium über Sprachwandel und seine bestimmenden Faktoren vom 30.9. - 2.10.1987 an der Universität Essen [a cura di Norbert Boretzky / Werner Enninger / Thomas Stolz], Bochum: Brockmeyer, pp. 289-316. 


\section{Regularly irregular - about the allomorphy of high-frequency Italian and French verbs}

Studies especially referring to Germanic languages (e.g. Nübling 2000) show that irregularity in the paradigms of highly frequent verbs such as auxiliaries, modal or movement verbs is due to some regular processes (sound and morphological changes) leading to distinctive forms in the respective paradigms; the development of the forms seem to reflect the underlying concept of relevance hierarchy as proposed by Bybee (1985). This concept means a correlation between morphological development and token frequency while type frequency is responsible for the adoption of morphological schemas, patterns or morphomes. The article tries to get a general idea of the applicability of the concept and the results of Nübling's study with regard to a couple of the most common verbs in French and Italian, namely Fr. aller/It. andare, Fr. venir/It. venire and old/middle Fr. ester (vs. estre $>$ mod. Fr. être)/It. stare.

Key words: morphology, allomorphy, analogy, irregularity, frequency 
